\title{
Previously diagnosed influenza infections and the risk of developing epilepsy
}

\author{
J. C. WILSON ${ }^{1 *}$, S. TOOVEY ${ }^{2}$, S. S. JICK ${ }^{3}$ AND C. R. MEIER ${ }^{1,3,4}$ \\ ${ }^{1}$ Basel Pharmacoepidemiology Unit (BPU), Division of Clinical Pharmacy and Epidemiology, Department of \\ Pharmaceutical Sciences, University of Basel, Switzerland \\ ${ }^{2}$ Division of Infection and Immunity, Royal Free and University College Medical School, Academic Centre for \\ Travel Medicine and Vaccines, London, UK \\ ${ }^{3}$ Boston Collaborative Drug Surveillance Program, Boston University School of Public Health, Lexington, MA, \\ USA \\ ${ }^{4}$ University Hospital Basel, Switzerland
}

Received 17 July 2014; Final revision 6 November 2014; Accepted 19 November 2014; first published online 18 December 2014

\section{SUMMARY}

Several epidemiological studies suggest a possible involvement of viral infection in the development of epilepsy. While recent research from in vitro studies increasingly supports the role of herpes simplex virus type 1 (HSV-1) in the pathogenesis of epilepsy, little is known about the role of other viral infections such as influenza. Using data from the Clinical Practice Research Datalink (CPRD), we conducted a matched case-control analysis to assess the association between GP-diagnosed influenza infections and the risk of developing an incident diagnosis of epilepsy. During the study period 11244 incident epilepsy cases and 44976 matched control patients were identified. Prior exposure to influenza was reported in $7 \cdot 5 \%$ of epilepsy cases and $6 \cdot 7 \%$ of controls [adjusted odds ratio (aOR) $1 \cdot 12,95 \%$ confidence interval (CI) $1 \cdot 03-1 \cdot 22$ ]. Prior history of 'complicated influenza', i.e. influenza associated with a possible super-infection, was associated with a slightly increased epilepsy risk (aOR $1 \cdot 64,95 \%$ CI $1 \cdot 10-2 \cdot 46$ ), particularly if recorded within the 2 months preceding the epilepsy diagnosis (aOR 6.03, 95\% CI $1 \cdot 10-33 \cdot 2$ ). Our findings suggest that prior influenza exposure does not appear to materially alter the risk of developing epilepsy. By contrast, influenza episodes accompanied by complications were associated with a slightly increased epilepsy risk.

Key words: Case-control, epilepsy, influenza.

\section{INTRODUCTION}

Epilepsy is a chronic neurological condition which is characterized by the occurrence of repeated seizures [1]. Globally, it is estimated that there are 50 million people

\footnotetext{
* Author for correspondence: Dr J. C. Wilson, Basel Pharmacoepidemiology Unit, Division of Clinical Pharmacy and Epidemiology, Department of Pharmaceutical Sciences, University of Basel, Formonterhof, St. Johanns-Vorstadt 27, CH-4031 Basel, Switzerland.

(Email: jessica.wilson@usb.ch)
}

living with epilepsy [1]. In the UK alone, about 600000 people have a diagnosis of epilepsy or receive antiepileptic drugs [2]. The main aetiological factors for epilepsy include genetic susceptibility, medical disorders such as stroke and other vascular diseases, dementia, head trauma, certain developmental disorders, and a history of febrile seizures during childhood [3].

In addition to the aetiological factors mentioned above, there is evidence to suggest that viral infections, such as herpes simplex virus (HSV), may play a role in the development of epilepsy [4-8]. Viral 
infections can result in the occurrence of viral encephalitis, which has been reported to increase the risk of developing epilepsy by 16-fold [5]. It has been noted that among survivors of HSV encephalitis (HSE), epilepsy is a major problem [4]. An observational study that assessed the long-term outcome of 42 patients diagnosed with herpes simplex encephalitis found that $24 \%$ of the 34 surviving patients had a later diagnosis of epilepsy [9]. More recent in vitro studies of the neuropathogenesis of HSV-associated seizures have shown that HSV-1 infection can lead to epileptiform activity and increased seizure susceptibility. This is thought to be through a direct change to the excitability of the hippocampal CA3 neuronal network and neuron loss, and a subsequent increase in synaptic reorganization in the supragranular area $[4,10,11]$.

While there is increasing research and understanding into the role of HSV infection and its association with epilepsy, few studies have examined the role of other viral infections such as influenza. A link between influenza and the development of neurological complications is widely recorded [12-14]. Several case series on the development of neurological complications following influenza reported a high frequency of both seizures and encephalopathy [13,15-18]. The majority of seizures appeared to be febrile, and a growing number of observational studies suggest an association between the occurrence of febrile seizures and the later development of epilepsy [19-22]. In addition, hemiconvulsions and transient periodic lateralized epileptiform discharges, which are associated with epilepsy [23], have been associated with influenza B-associated encephalopathy [24].

While recent studies support the involvement of HSV-1 infection in the development of epilepsy, the role of other viral infections such as influenza remains largely unclear. In these circumstances, further research into whether an association exists between influenza and the risk of epilepsy would be beneficial. Therefore, we set out to conduct a case-control analysis using data from the Clinical Practice Research Datalink (CPRD), to assess whether influenza episodes are associated with an altered risk of developing an incident diagnosis of epilepsy.

\section{METHODS}

\section{Data source}

The CPRD provides anonymized healthcare information on some 8 million patients in the UK, going back as far as 1987. Information on demographics (age, sex, weight, height), consultations, symptoms, diagnoses, specialist referrals as well as details on prescribed medications and lifestyle factors (e.g. tobacco and alcohol use) are recorded in the CPRD by specially trained General Practitioners (GPs). Read and Multilex codes are used to classify medical diagnoses and drug prescriptions, respectively. Recorded information on diagnoses and drug exposure has been validated and proven to be of high quality $[25,26]$. The CPRD has been previously used for studies on influenza [27-30]. The study protocol was reviewed and approved by the Independent Scientific Advisory Committee (ISAC) for Medicines and Healthcare Products Regulatory Agency Database Research.

\section{Study population}

Cases were defined as patients aged $\leqslant 90$ years with a first-time recorded Read code for epilepsy, seizures, convulsions or fits (subsequently referred to as 'epilepsy'), followed by at least $\geqslant 2$ repeat prescriptions for an anti-epileptic medication during the period of 1 January 1995 to 30 July 2012. The index date was defined as the date of the first recorded diagnosis of epilepsy. We excluded all cases with $<3$ years of recorded history, as well as those with a recording for cancer (excluding non-melanoma skin cancers), developmental disorders (i.e. Down syndrome, cerebral palsy), HIV/AIDS, alcoholism or drug abuse prior to the index date.

Four control subjects were randomly identified for each epilepsy case patient, matched to cases on index date, GP practice, year of birth, gender, and number of years of previous recorded history in the database. Control patients were defined as patients with no recorded history of epilepsy, seizures, convulsions, or fits, and no recorded prescription for antiepileptic medication prior to the index date. The same exclusion criterion was applied to controls as to cases.

\section{Assessment of influenza}

Using Read-coded information recorded in the patient record prior to the index date, the number of influenza episodes and the timing of the last influenza episode prior to the index date were assessed. Patients who ever had an influenza or influenza-like illness (ILI) diagnosis recorded prior to the index date were stratified by number of previous recorded infections $(1,2, \geqslant 3$ episodes) and by timing of previous influenza episodes 
(last recorded episode recorded within 1-59, 60-364, $365-729$, or $\geqslant 730$ days prior to the index date). If a patient had more than one influenza episode recorded, these were considered two separate influenza episodes if recorded $>30$ days apart. Of those who ever had influenza recorded by the GP prior to the index date, we further assessed whether any of these previous influenza episodes were followed within 30 days by a recording for a clinical complication, based on a possible bacterial superinfection; these included sepsis, meningitis, encephalitis, or pneumonia. An influenza episode accompanied by such a recording may reflect a more severe influenza, and were referred to as 'influenza with complications'. Bacterial superinfections are noted to be a common cause of influenza-related hospitalization [31].

\section{Assessment of other exposures}

The prevalence of various comorbidities and co-medications was assessed for all cases and controls. A patient was coded as having a comorbidity or medication use if there was a code for that diagnosis or drug in the patient record any time prior to the index date. For other covariates including body mass index (BMI $\leqslant 18 \cdot 4,18 \cdot 5-24 \cdot 9,25-29 \cdot 9$, $\geqslant 30$ $\mathrm{kg} / \mathrm{m}^{2}$ ), smoking (never, ex-smoker, current, unknown), and alcohol use (never, ex-drinker, current, unknown), information recorded in the patient record closest prior to the index date was used. In addition, the following covariates were assessed to test for potential confounding: asthma, chronic obstructive pulmonary disease (COPD), ischaemic heart disease, heart failure, atrial fibrillation, stroke or transient ischaemic attack (TIA), hypertension, hypercholesterolaemia, diabetes mellitus, receipt of influenza vaccinations, antibiotic medication, systemic corticosteroids, and immunosuppressant drugs.

\section{Statistical analysis}

Conditional logistic regression analyses were conducted to explore the association between influenza episodes prior to the index date and the risk of a first-time epilepsy diagnosis. Univariate and multivariate odds ratios (ORs) with $95 \%$ confidence intervals (CIs) were assessed for 'ever $v s$. never' exposure to influenza, as well as analyses stratified by frequency and timing of previous infections. Potential confounding factors were assessed and included in the model if there was $a \geqslant 10 \%$ change in the univariate estimate of epilepsy risk associated with influenza. In addition, interaction by gender was evaluated. All statistical analyses were conducted using SAS release 9.3 (SAS Institute Inc., USA).

\section{RESULTS}

During the study period 11244 epilepsy cases were identified and matched to 44976 control patients ( $49 \cdot 7 \%$ female). Over $67 \%$ of cases were aged $<60$ years at the index date. Characteristics for both cases and controls are shown in Table 1. A substantially increased risk of epilepsy was observed in individuals with a prior history of stroke and dementia (Table 1). The majority ( $89 \%$ ) of influenza episodes appeared to be seasonal, i.e. recorded during the period October through to April.

There was no increased risk of developing epilepsy in patients with one or more influenza episodes at any time prior to the index date compared to those with no recorded influenza (OR $1 \cdot 12,95 \%$ CI $1 \cdot 03$ $1 \cdot 22$ ) after adjusting for smoking status, alcohol use, history of stroke, and prior use of corticosteroids or antibiotics. There was no material effect of timing of last influenza prior to the index date or by number of prior episodes (Table 2). Tests for interaction by gender revealed no evidence of effect modification $(P=0.1311)$. Stratification by gender yielded an adjusted OR (aOR) of $1.20(95 \%$ CI $1.06-1 \cdot 36)$ for males, and of $1.05(95 \%$ CI $0 \cdot 93-1 \cdot 19)$ for females.

For patients with 'complicated influenza', over $97 \%$ had a subsequent diagnosis of pneumonia. An increased risk of developing epilepsy with prior 'complicated influenza' exposure was observed in both the crude (OR 1·75, 95\% CI 1·19-2.56) and adjusted (OR $1 \cdot 64,95 \%$ CI 1·10-2.46) analyses. In Table 3 we present the effects stratified by timing of the last recorded influenza episode. There was a substantially increased risk of epilepsy for those with a recent (within 60 days) 'complicated influenza' compared to patients with no history of influenza (aOR 6.03, 95\% CI 1·10-33·2). This finding, however, was based on only three cases and three controls. A significant trend with increasing time since last influenza episode was observed; however numbers in each category were small (Table 3 ). For most cases with a 'complicated influenza' the last diagnosis was $\geqslant 730$ days prior to the index date and there was an increase in risk in these cases (aOR $1 \cdot 94,95 \%$ CI $1 \cdot 22-3 \cdot 11$ ). The majority $(97 \%)$ of these patients had only one 'complicated influenza' so we could not evaluate the effects of increasing 
Table 1. Characteristics of epilepsy cases and their matched controls

\begin{tabular}{|c|c|c|c|}
\hline & Cases $(n=11244)$ & Controls $(n=44976)$ & OR $(95 \% \mathrm{CI})$ \\
\hline \multicolumn{4}{|l|}{ Gender } \\
\hline Male & $5652(50 \cdot 3)$ & $22608(50 \cdot 3)$ & - \\
\hline Female & $5592(49 \cdot 7)$ & $22368(49 \cdot 7)$ & - \\
\hline \multicolumn{4}{|l|}{ Age, years } \\
\hline $0-9$ & $1257(11 \cdot 2)$ & $5079(11 \cdot 3)$ & - \\
\hline $10-19$ & $2249(20 \cdot 0)$ & $8958(19 \cdot 9)$ & - \\
\hline $20-29$ & $1609(14 \cdot 3)$ & $6418(14 \cdot 3)$ & - \\
\hline $30-39$ & $499(4 \cdot 4)$ & $1987(4 \cdot 4)$ & - \\
\hline $40-49$ & $1456(13 \cdot 0)$ & $5829(13 \cdot 0)$ & - \\
\hline $50-59$ & $486(4 \cdot 3)$ & $1933(4 \cdot 3)$ & - \\
\hline $60-69$ & $1150(10 \cdot 2)$ & $4637(10 \cdot 3)$ & - \\
\hline$\geqslant 70$ & $2538(22 \cdot 6)$ & $10135(22 \cdot 5)$ & - \\
\hline \multicolumn{4}{|l|}{ Body mass index $\left(\mathrm{kg} / \mathrm{m}^{2}\right)$} \\
\hline$\leqslant 18 \cdot 4$ & $220(2 \cdot 0)$ & $631(1 \cdot 1)$ & $1 \cdot 38(1 \cdot 17-1 \cdot 62)$ \\
\hline $18 \cdot 5-24 \cdot 9$ & $2670(23 \cdot 8)$ & $10474(23 \cdot 3)$ & $1 \cdot 00$ \\
\hline $25-29 \cdot 9$ & $2077(18 \cdot 5)$ & $8654(19 \cdot 2)$ & $0.94(0.88-1 \cdot 00)$ \\
\hline$\geqslant 30$ & $1155(10 \cdot 3)$ & $4448(9 \cdot 9)$ & $1.02(0.94-1 \cdot 10)$ \\
\hline Unknown & $5122(45 \cdot 6)$ & $20769(46 \cdot 2)$ & $0.94(0 \cdot 87-1 \cdot 01)$ \\
\hline \multicolumn{4}{|l|}{ Smoking status } \\
\hline Non-smoker & $3934(35 \cdot 0)$ & $16261(36 \cdot 2)$ & $1 \cdot 00$ \\
\hline Current smoker & $1765(15 \cdot 7)$ & $6199(13 \cdot 8)$ & $1 \cdot 19(1 \cdot 12-1 \cdot 27)$ \\
\hline Ex-smoker & $1685(15 \cdot 0)$ & $6527(14 \cdot 5)$ & $1.09(1.02-1 \cdot 17)$ \\
\hline Unknown & $3860(34 \cdot 3)$ & $15989(35 \cdot 6)$ & $0.91(0.84-0.98)$ \\
\hline \multicolumn{4}{|l|}{ Alcohol use } \\
\hline Non-drinker & $1647(14 \cdot 7)$ & $5412(12 \cdot 0)$ & $1 \cdot 00$ \\
\hline Current drinker & $5114(45 \cdot 5)$ & $20946(46 \cdot 6)$ & $0.79(0.74-0.85)$ \\
\hline Former drinker & $163(1 \cdot 6)$ & $327(0 \cdot 7)$ & $1 \cdot 68(1 \cdot 38-2 \cdot 05)$ \\
\hline Unknown & $4320(38 \cdot 4)$ & $18291(40 \cdot 7)$ & $0.68(0.63-0.74)$ \\
\hline \multicolumn{4}{|l|}{ Risk factors } \\
\hline Diabetes & $577(5 \cdot 1)$ & $1854(4 \cdot 1)$ & $1 \cdot 28(1 \cdot 16-1 \cdot 41)$ \\
\hline Arrhythmia & $666(5 \cdot 9)$ & $1747(3 \cdot 9)$ & $1.63(1.48-1.80)$ \\
\hline Heart failure & $258(2 \cdot 3)$ & $833(1 \cdot 9)$ & $1 \cdot 27(1 \cdot 10-1 \cdot 48)$ \\
\hline Ischaemic heart disease & $776(6 \cdot 9)$ & $2814(6 \cdot 3)$ & $1 \cdot 13(1 \cdot 04-1 \cdot 24)$ \\
\hline Hypertension & $2192(19 \cdot 5)$ & $7561(16 \cdot 8)$ & $1 \cdot 31(1 \cdot 23-1 \cdot 40)$ \\
\hline Stroke & $1774(15 \cdot 8)$ & $1343(3 \cdot 0)$ & $8 \cdot 61(7 \cdot 87-9 \cdot 42)$ \\
\hline Pulmonary embolism & $98(0 \cdot 9)$ & $240(0 \cdot 5)$ & $1 \cdot 65(1 \cdot 30-2 \cdot 09)$ \\
\hline Deep vein thrombosis & $158(1 \cdot 4)$ & $419(0 \cdot 9)$ & $1 \cdot 52(1 \cdot 27-1 \cdot 84)$ \\
\hline Cholesterol & $783(7 \cdot 0)$ & $2744(6 \cdot 1)$ & $1 \cdot 19(1 \cdot 08-1 \cdot 30)$ \\
\hline Dementia & $409(3 \cdot 6)$ & $261(0 \cdot 6)$ & $8 \cdot 21(6 \cdot 88-9 \cdot 80)$ \\
\hline \multicolumn{4}{|l|}{ Prior medication use } \\
\hline Systemic corticosteroids & $1882(16 \cdot 7)$ & $6559(14 \cdot 6)$ & $1 \cdot 20(1 \cdot 13-1 \cdot 27)$ \\
\hline Immunosuppressant & $106(0 \cdot 9)$ & $267(0 \cdot 6)$ & $1 \cdot 60(1 \cdot 28-2 \cdot 01)$ \\
\hline Antibiotics & $9556(85 \cdot 0)$ & $37271(82 \cdot 9)$ & $1 \cdot 23(1 \cdot 15-1 \cdot 31)$ \\
\hline
\end{tabular}

OR, Odds ratio; CI, confidence interval.

number of such episodes. A direct comparison between 'complicated influenza' and 'influenza' yielded an aOR of $2 \cdot 08$ (95\% CI $0 \cdot 81-5 \cdot 34)$.

\section{DISCUSSION}

There have been reports of neurological effects associated with influenza infections, and an increase in seizures and epilepsy reported in both children and adults following the 2009 influenza A(H1N1) pandemic $[14,32]$. The exact biological mechanism by which severe influenza may lead to epilepsy, however, is uncertain. Research has shown that viral infections can induce neuronal inflammation and pro-inflammatory cytokine release, leading to an influx of pro-inflammatory mediators into the CNS 
Table 2. Unadjusted and adjusted odds ratios for the association between epilepsy and previous influenza episodes

\begin{tabular}{|c|c|c|c|c|}
\hline & $\begin{array}{l}\text { Cases } \\
(n=11244)\end{array}$ & $\begin{array}{l}\text { Controls } \\
(n=44976)\end{array}$ & $\begin{array}{l}\text { Unadjusted } \\
\text { OR }(95 \% \mathrm{CI})\end{array}$ & $\begin{array}{l}\text { Adjusted } \dagger \\
\text { OR }(95 \% \text { CI })\end{array}$ \\
\hline \multicolumn{5}{|l|}{ Influenza } \\
\hline Never & $10404(92 \cdot 5)$ & $41957(93 \cdot 3)$ & $1 \cdot 0$ & $1 \cdot 0$ \\
\hline Ever & $840(7 \cdot 5)$ & $3019(6 \cdot 7)$ & $1 \cdot 13(1 \cdot 04-1 \cdot 23)$ & $1 \cdot 12(1 \cdot 03-1 \cdot 22)^{*}$ \\
\hline \multicolumn{5}{|c|}{ Number of influenza episodes } \\
\hline 0 & $10404(92 \cdot 5)$ & $41957(93 \cdot 3)$ & $1 \cdot 0$ & $1 \cdot 0$ \\
\hline 1 & $731(6 \cdot 5)$ & $2619(5 \cdot 8)$ & $1 \cdot 14(1 \cdot 04-1 \cdot 24)$ & $1 \cdot 13(1 \cdot 03-1 \cdot 24)^{*}$ \\
\hline 2 & $92(0 \cdot 8)$ & $316(0 \cdot 7)$ & $1 \cdot 19(0 \cdot 94-1 \cdot 51)$ & $1 \cdot 10(0 \cdot 86-1 \cdot 41)$ \\
\hline$\geqslant 3$ & $17(0 \cdot 2)$ & $84(0 \cdot 2)$ & $0 \cdot 82(0 \cdot 47-1 \cdot 43)$ & $0 \cdot 79(0 \cdot 43-1 \cdot 44)$ \\
\hline$P_{\text {trend }}$ & & & & $0 \cdot 0420$ \\
\hline \multicolumn{5}{|c|}{ Timing of last influenza episode, days } \\
\hline 0 & $10404(92 \cdot 5)$ & $41957(93 \cdot 3)$ & $1 \cdot 0$ & $1 \cdot 0$ \\
\hline $1-59$ & $16(0 \cdot 1)$ & $62(0 \cdot 1)$ & $1 \cdot 13(0 \cdot 72-1 \cdot 78)$ & $1 \cdot 17(0 \cdot 65-2 \cdot 01)$ \\
\hline $60-364$ & $79(0 \cdot 7)$ & $237(0 \cdot 5)$ & $1 \cdot 36(1.04-1 \cdot 78)$ & $1 \cdot 30(0 \cdot 98-1 \cdot 73)$ \\
\hline $365-729$ & $88(0 \cdot 8)$ & $301(0 \cdot 7)$ & $1.19(0.94-1.52)$ & $1 \cdot 15(0 \cdot 89-1 \cdot 48)$ \\
\hline$\geqslant 730$ & $657(5 \cdot 8)$ & $2419(5 \cdot 4)$ & $1 \cdot 11(1 \cdot 01-1 \cdot 21)$ & $1 \cdot 10(0 \cdot 99-1 \cdot 21)$ \\
\hline$P_{\text {trend }}$ & & & & $0 \cdot 0242$ \\
\hline
\end{tabular}

OR, Odds ratio; CI, confidence interval.

$\dagger$ Adjusted for smoking status (non, current, ex, unknown), alcohol use (non, current, ex, unknown), history of stroke, prior use of systemic corticosteroids, prior use of antibiotics (all recorded prior to index date).

$\$$ Prior to index date.

$*<0 \cdot 05$ significance.

Table 3. Unadjusted and adjusted odds ratios for the association between epilepsy and previous 'complicated influenza' episodes

\begin{tabular}{|c|c|c|c|c|}
\hline & $\begin{array}{l}\text { Cases } \\
(n=10442)\end{array}$ & $\begin{array}{l}\text { Controls } \\
(n=42044)\end{array}$ & $\begin{array}{l}\text { Unadjusted } \\
\text { OR }(95 \% \mathrm{CI})\end{array}$ & $\begin{array}{l}\text { Adjusted } \dagger \\
\text { OR }(95 \% \text { CI })\end{array}$ \\
\hline \multicolumn{5}{|c|}{ Complicated influenza followed by clinical complications } \\
\hline Never & $10404(99 \cdot 6)$ & $41957(99 \cdot 8)$ & $1 \cdot 0$ & $1 \cdot 0$ \\
\hline$\geqslant 1$ & $38(0 \cdot 4)$ & $87(0 \cdot 2)$ & $1 \cdot 75(1 \cdot 19-2 \cdot 56)$ & $1 \cdot 64(1 \cdot 10-2 \cdot 46)^{*}$ \\
\hline \multicolumn{5}{|c|}{ Last complicated influenza episode, dayst } \\
\hline 0 & $10404(99 \cdot 6)$ & $41957(99 \cdot 8)$ & $1 \cdot 0$ & $1 \cdot 0$ \\
\hline $1-59$ & $3(0 \cdot 03)$ & $3(0 \cdot 01)$ & $4 \cdot 00(0 \cdot 81-19 \cdot 82)$ & $6 \cdot 03(1 \cdot 10-33 \cdot 20)^{*}$ \\
\hline $60-364$ & $3(0 \cdot 03)$ & $11(0 \cdot 03)$ & $1.09(0 \cdot 30-3 \cdot 91)$ & $0 \cdot 77(0 \cdot 20-2 \cdot 98)$ \\
\hline $365-729$ & $4(0 \cdot 04)$ & $15(0 \cdot 04)$ & $1 \cdot 07(0 \cdot 35-3 \cdot 21)$ & $0 \cdot 74(0 \cdot 22-2 \cdot 43)$ \\
\hline$\geqslant 730$ & $28(0 \cdot 3)$ & $58(0 \cdot 14)$ & $1.93(1.23-3.03)$ & $1 \cdot 94(1 \cdot 22-3 \cdot 11)^{*}$ \\
\hline$P_{\text {trend }}$ & & & & $0 \cdot 0127$ \\
\hline
\end{tabular}

OR, Odds ratio; CI, confidence interval.

$\dagger$ Adjusted for smoking status (non, current, ex, unknown), alcohol use (non, current, ex, unknown), history of stroke, prior use of systemic corticosteroids, prior use of antibiotics (all recorded prior to index date).

$\$$ Prior to index date.

$*<0.05$ significance.

$[33,34]$. It has been suggested that neuroinflammation can create the conditions for a faster than normal rate of neuronal degeneration, with frequent, sustained, or severe periods of non-symptomatic virus driven neuroinflammation, heightening an individual's proclivity to subsequent brain dysfunctions as they grow older
[34]. A study by Jurgens et al., which examined the impact of influenza on the hippocampus and on cognition, found evidence to suggest that peripheral influenza infection may induce neuroinflammation, increase microglial reactivity, and alter the morphology of the hippocampal region [35]. Experimental studies 
indicate glial cells and pro-inflammatory mediators play an important role in the pathophysiology of epilepsy [33, 36-38], with the activation of microglial cells resulting in a decrease in the seizure threshold and increased neuronal stimulation through the release of pro-inflammatory molecules [34].

The present large nested case-control study provides little evidence for an association between influenza exposure and epilepsy, with a reported overall OR close to 1 (aOR $1 \cdot 12,95 \%$ CI $1 \cdot 03-1 \cdot 22$ ). Nor was there an association with increased frequency of influenza episodes or timing of influenza. By contrast, any history of 'complicated influenza' episodes followed by additional bacterial infections (i.e. mainly pneumonia) yielded an increased risk of developing epilepsy (aOR $1 \cdot 64,95 \%$ CI $1 \cdot 10-2 \cdot 46$ ), which was particularly pronounced if the infection occurred within 2 months of the epilepsy diagnosis (aOR $6 \cdot 03,95 \%$ CI $1 \cdot 10$ $33 \cdot 2$ ), and which was elevated for a considerable period of time after the influenza exposure (aOR 1.94, $95 \%$ CI $1 \cdot 22-3 \cdot 11$ ) for an infection $\geqslant 730$ days prior to the index date. However, it is important to note that the numbers in these analyses were small, and we cannot fully rule out the possibility of chance despite the statistical significance of the findings. In addition, we cannot rule out the possible presence of some residual confounding. Consequently, the results should be interpreted with caution. Moreover, the majority of 'complicated influenza' cases included individuals with a subsequent diagnosis of pneumonia. Aspiration pneumonia as a result of the inhalation of food particles during seizures has been reported as a possible complication in epileptic patients [39]. It is thus possible that this observed increase in risk may be a result of pneumonia in patients with undiagnosed epilepsy, which then lead to an epilepsy diagnosis after an episode of aspiration pneumonia. Although, a significant increase in epilepsy risk following 'complicated influenza' was also seen $\geqslant 2$ years after an influenza episode, where this notion of reverse causality seems to be unlikely. Even though an observational study like the current one cannot prove causality, this finding may reflect a causal association between 'complicated influenza' and epilepsy, as it has been shown that pandemic 2009 influenza A (H1N1)-associated pneumonia did result in increased influenza viral concentrations, reduced viral clearance and up-regulated plasma pro-inflammatory cytokine responses [40].

This large case-control study utilized data from the CPRD, one of the largest and best-validated databases available, to examine the association between influenza infection and epilepsy risk. As it has been documented that a misdiagnosis of epilepsy can occur in about $20-30 \%$ of patients [2], we sought to avoid possible misclassification of epilepsy cases by applying a strict outcome definition and included only those patients who had, in addition to the epilepsy diagnosis, at least two repeat prescriptions for an anti-epileptic medication. An additional strength of this study is the ability to account for a variety of potential confounders including antibiotic use, corticosteroid and immunosuppressant use, and a prior history of stroke. All patients included in this study had at least 3 years of data prior to their index date.

It is also important to highlight potential limitations of the study. First, GP-diagnosed influenza is identified mainly through clinical diagnosis and is generally not supported by a viral test. Therefore, we cannot rule out the possibility that some recorded influenza diagnoses were not a consequence of the influenza virus, but rather as a result of an alternative infectious agent causing an 'influenza-like' illness. Furthermore, as influenza may be managed or treated at home without GP involvement, it is likely that some influenza episodes were not recorded by the GP. However, a study published in 2000 by our group found that the rate of recorded influenza infections over a 6-year period was closely similar to those derived from the UK sentinel system, the gold standard for UK influenza assessment at the time [27]. Additionally, $89 \%$ of all last-recorded influenza episodes were seasonal occurring between October and April. Hence, the possible misclassification of influenza episodes is likely to be limited. The clinical complications used to define 'complicated influenza' were based on possible bacterial superinfections (i.e. a subsequent diagnosis of sepsis, pneumonia, meningitis or encephalitis), without laboratory results to confirm a bacterial presence. Even though such complications may also be viral, it has been reported that secondary bacterial infections during and shortly after influenza virus infection recovery are a more common cause of pneumonia [31]. A further limitation is that the diagnosis of influenza and of epilepsy might not be recorded on the same day that the diagnosis was made, so that the analysis of the timing of the last-recorded influenza infection and the occurrence of epilepsy might not be highly accurate. Thus, it is possible that the few cases contributing to the substantially increased epilepsy risk for patients with a complicated influenza within 60 days before the 
index date may have been febrile seizures. However, it is unlikely that the observed increase in epilepsy risk associated with 'complicated influenza' for those who developed epilepsy $\geqslant 2$ years after the last-recorded influenza episode is a result of misdiagnosed influenza-associated febrile seizures. Furthermore, over $68 \%$ of epilepsy cases were reported in adult patients in whom febrile seizures are less likely to occur than in children.

In conclusion, this large case-control study found little evidence for an association between influenza exposure and epilepsy risk overall. However, we observed an increased risk of epilepsy in individuals with a history of 'complicated influenza', which was still present 2 years following exposure.

\section{ACKNOWLEDGEMENTS}

The authors thank BPU data programmer Mr Pascal Egger for his important contribution to the study. This work was funded by an unconditional grant from Hoffmann-La Roche Ltd, Switzerland.

\section{DECLARATION OF INTEREST}

None.

\section{REFERENCES}

1. World Health Organisation. Epilepsy: Fact sheet No. 999, 2012 (http://www.who.int/mediacentre/factsheets/ fs999/en/). Accessed 12 February 2013.

2. JEC. Epilepsy prevalence, incidence and other statistics. Leeds, UK: Joint Epilepsy Council (JEC), 2011.

3. Adamolekun B. Seizure disorders, 2013 (http://www. merckmanuals.com/professional/neurologic_disorders/ seizure_disorders/seizure_disorders.html). Accessed 8 February 2013.

4. Sellner J, Trinka E. Seizures and epilepsy in herpes simplex virus encephalitis: current concepts and future directions of pathogenesis and management. Journal of Neurology 2012; 259: 2019-2030.

5. Sander JW. Infectious agents and epilepsy. In: Knobler $\mathrm{SL}$, et al. eds. The Infectious Etiology of Chronic Diseases: Defining the Relationship, Enhancing the Research, and Mitigating the Effects-Workshop Summary (2004). Washington: National Academies Press, 2004, pp. 93-99.

6. Misra UK, Tan CT, Kalita J. Viral encephalitis and epilepsy. Epilepsia 2008; 49 (Suppl. 6): 13-18.

7. Pradhan S, Yadav R. Seizures and epilepsy in central nervous system infections. Neurology Asia 2004; 9: 4-9.

8. Michael BD, Solomon T. Seizures and encephalitis: Clinical features, management, and potential pathophysiologic mechanisms. Epilepsia 2012; 53 (Suppl. 4): 63-71.
9. McGrath N, et al. Herpes simplex encephalitis treated with acyclovir: diagnosis and long term outcome. Journal of Neurology, Neurosurgery, and Psychiatry 1997; 63: 321-326.

10. Chen SF, et al. Seizure, neuron loss, and mossy fiber sprouting in herpes simplex virus type 1-infected organotypic hippocampal cultures. Epilepsia 2004; 45: 322-332.

11. Wu HM, et al. Herpes simplex virus type 1 inoculation enhances hippocampal excitability and seizure susceptibility in mice. European Journal of Neuroscience 2003; 18: 3294-3304.

12. Ekstrand JJ. Neurologic complications of influenza. Seminars in Pediatric Neurology 2012; 19: 96-100.

13. Toovey S. Influenza-associated central nervous system dysfunction: a literature review. Travel Medicine and Infectious Disease 2008; 6: 114-124.

14. Ekstrand JJ, et al. Heightened neurologic complications in children with pandemic H1N1 influenza. Annals of Neurology 2010; 68: 762-766.

15. Chiu $\mathbf{S}$, et al. Influenza $A$ infection is an important cause of febrile seizures. Pediatrics 2001; 108: E63.

16. Chung B, Tsang A, Wong V. Neurologic complications in children hospitalized with influenza: comparison between USA and Hong Kong. Journal of Pediatrics 2007; 151: e17-18.

17. Surana P, et al. Neurological complications of pandemic influenza A H1N 12009 infection: European case series and review. European Journal of Pediatrics 2011; 170: 1007-1015.

18. Landau Y, et al. Pediatric neurologic complications associated with influenza A H1N1. Pediatric Neurology 2011; 44: 47-51.

19. Annegers JF, et al. The risk of epilepsy following febrile convulsions. Neurology 1979; 29: 297-303.

20. Berg AT, Shinnar S. Unprovoked seizures in children with febrile seizures: short-term outcome. Neurology 1996; 47: 562-568.

21. Dube CM, et al. Fever, febrile seizures and epilepsy. Trends in Neurosciences 2007; 30: 490-496.

22. Vestergaard M, et al. The long-term risk of epilepsy after febrile seizures in susceptible subgroups. American Journal of Epidemiology 2007; 165: 911-918.

23. Kumar S, Kesavalu N, Chandy E. Periodic lateralized epileptiform discharges in a child with solitary cysticercus granuloma. Neurology India 2004 52: 523-524.

24. Kurita A, et al. Periodic lateralized epileptiform discharges in influenza B-associated encephalopathy. Internal Medicine 2001; 40: 813-816.

25. Khan NF, Harrison SE, Rose PW. Validity of diagnostic coding within the General Practice Research Database: a systematic review. British Journal of General Practice 2010; 60: e128-136.

26. Herrett E, et al. Validation and validity of diagnoses in the General Practice Research Database: a systematic review. British Journal of Clinical Pharmacology 2010; 69: 4-14.

27. Meier CR, et al. Population-based study on incidence, risk factors, clinical complications and drug utilisation associated with influenza in the United Kingdom. 
European Journal of Clinical Microbiology \& Infectious Diseases 2000; 19: 834-842.

28. Jick H, Chamberlin DP, Hagberg KW. Vaccine policy and incidence of influenza. Epidemiology 2010; 21: 270-271.

29. Jick H, Hagberg KW. Effectiveness of influenza vaccination in the United Kingdom, 1996-2007. Pharmacotherapy 2010; 30: 1199-1206.

30. Toovey S, Jick SS, Meier CR. Parkinson's disease or Parkinson symptoms following seasonal influenza. Influenza and Other Respiratory Viruses 2011; 5: 328-333.

31. Van Der Sluijs KF, et al. Bench-to-bedside review: Bacterial pneumonia with influenza - pathogenesis and clinical implications. Critical Care 2010; 14:219.

32. Yeo L, et al. Complex partial status epilepticus associated with adult H1N1 infection. Journal of Clinical Neuroscience 2012; 19: 1728-1730.

33. Cusick MF, et al. Infiltrating macrophages are key to the development of seizures following virus infection. Journal of Virology 2013; 87: 1849-1860.

34. Deleidi M, Isacson O. Viral and inflammatory triggers of neurodegenerative diseases. Science Translational Medicine 2012; 4: 121-123.
35. Jurgens HA, Amancherla K, Johnson RW. Influenza infection induces neuroinflammation, alters hippocampal neuron morphology, and impairs cognition in adult mice. Journal of Neuroscience 2012; 32: 3958 3968.

36. Libbey JE, et al. Interleukin-6, produced by resident cells of the central nervous system and infiltrating cells, contributes to the development of seizures following viral infection. Journal of Virology 2011; 85: 6913-6922.

37. Vezzani A, Balosso S, Ravizza T. The role of cytokines in the pathophysiology of epilepsy. Brain, Behavior, and Immunity 2008; 22: 797-803.

38. Vezzani A, et al. The role of inflammation in epilepsy. Nature Reviews Neurology 2011; 7: 31-40.

39. Li S, Ding D, Wu J. Definitions and epidemiology of epilepsy. In: Shorvon S, et al. eds. Oxford Textbook of Epilepsy and Epileptic Seizures. UK: Oxford University Press, 2013, pp. 51-60.

40. Lee $\mathbf{N}$, et al. Viral clearance and inflammatory response patterns in adults hospitalized for pandemic 2009 influenza $\mathrm{A}(\mathrm{H} 1 \mathrm{~N} 1)$ virus pneumonia. Antiviral Therapy 2011; 16: 237-247. 